\title{
Computing Multiplicities of Lie Group Representations
}

\author{
Matthias Christandl \\ Department of Physics \\ ETH Zürich \\ Zürich, Switzerland \\ Email: christandl@phys.ethz.ch
}

\author{
Brent Doran \\ Department of Mathematics \\ ETH Zürich \\ Zürich, Switzerland \\ Email: brent.doran@math.ethz.ch
}

\author{
Michael Walter \\ Department of Physics \\ ETH Zürich \\ Zürich, Switzerland \\ Email: mwalter@itp.phys.ethz.ch
}

\begin{abstract}
For fixed compact connected Lie groups $\mathbf{H} \subseteq \mathbf{G}$, we provide a polynomial time algorithm to compute the multiplicity of a given irreducible representation of $H$ in the restriction of an irreducible representation of G. Our algorithm is based on a finite difference formula which makes the multiplicities amenable to Barvinok's algorithm for counting integral points in polytopes.

The Kronecker coefficients of the symmetric group, which can be seen to be a special case of such multiplicities, play an important role in the geometric complexity theory approach to the $P$ vs. NP problem. Whereas their computation is known to be \#P-hard for Young diagrams with an arbitrary number of rows, our algorithm computes them in polynomial time if the number of rows is bounded. We complement our work by showing that information on the asymptotic growth rates of multiplicities in the coordinate rings of orbit closures does not directly lead to new complexity-theoretic obstructions beyond what can be obtained from the moment polytopes of the orbit closures. Nonasymptotic information on the multiplicities, such as provided by our algorithm, may therefore be essential in order to find obstructions in geometric complexity theory.
\end{abstract}

\section{INTRODUCTION}

The decomposition of Lie group representations into irreducible sub-representations is a fundamental problem of mathematics with a variety of applications to the sciences. In atomic and molecular physics (Clebsch-Gordan series), as well as in high-energy physics, this problem has been studied extensively [1-3], perhaps most famously in Ne'eman and Gell-Mann's eight-fold way of elementary particles [4-6]. In pure mathematics, the combinatorial resolution of the problem of decomposing tensor products of irreducible representations of the unitary group by Knutson and Tao has been a recent highlight with a long history of research [7, 8]. More recently, the theories of quantum information [9-11], computation and complexity [12], as well as the geometric complexity theory approach to the $\mathbf{P}$ vs. NP problem [13-15] have brought the representation theory of Lie groups to the attention of the computer science community.

In this paper, we study the problem of computing multiplicities of Lie group representations:

Problem I.1 (Subgroup Restriction Problem). Let $f: H \rightarrow G$ be a homomorphism between compact connected Lie groups $H$ and $G$. The subgroup restriction problem for $f$ is to determine the multiplicity $m_{\mu}^{\lambda}$ of the irreducible $H$-representation $V_{H, \mu}$ in the irreducible $G$-representation $V_{G, \lambda}$ when given as input the highest weights $\mu$ and $\lambda$ (specified as bitstrings containing their coordinates with respect to fixed bases of fundamental weights, see $\$ V$.

The name subgroup restriction problem comes from the archetypical case where the map $f$ is induced by the inclusion of a subgroup $H \subseteq G$. Problem I.1 is also known as the branching problem. The main result of this paper is a polynomial-time algorithm for Problem I.1

Theorem 1.2. For any homomorphism $f: H \rightarrow G$ between compact connected Lie groups $H$ and $G$, there is a polynomial-time algorithm for the subgroup restriction problem for $f$.

Indeed, we describe a concrete algorithm Algorithm V.1. In particular, for any fixed $\lambda$ and $\mu$ the stretching function $k \mapsto m_{k \mu}^{k \lambda}$ can be evaluated in polynomial time.

Corollary I.3. For any homomorphism $f: H \rightarrow G$ between compact connected Lie groups $H$ and $G$, positivity of the coefficients $m_{\mu}^{\lambda}$ can be decided in polynomial time.

Mulmuley conjectures that deciding positivity of the multiplicities $m_{\mu}^{\lambda}$ is possible in polynomial time if the group homomorphism $f$ is also part of the input [16]. Corollary I.3 can be regarded as supporting evidence that this conjecture might in fact be true for general $f$ (note that for specific families of homomorphisms, such as those corresponding to the Littlewood-Richardson coefficients, positivity can be decided in polynomial time [8, 17]). However, any approach to deciding positivity that proceeds by computing the actual multiplicities is of course expected to fail, since the latter problem is well-known to be \#P-hard [18, 19].

We establish Theorem I.2 by deriving a novel formula for the multiplicities $m_{\mu}^{\lambda}$ (Theorem IV.2), which is obtained in three steps: First, we restrict from the group $G$ to its maximal torus $T_{G}$; the corresponding weight multiplicities can be computed efficiently by using the classical Kostant multiplicity formula [20, 21] or in fact by evaluating a single vector partition function [22-24] (\$II). Second, we restrict all weights to a maximal torus $T_{H}$ of $H$. Third, we recover the multiplicity of an irreducible $H$-representation by using 
a finite-difference formula (Proposition III.1). By carefully combining the first two steps, Problem I.1 can be reduced to counting integral points in certain rational convex polytopes of bounded dimension, which can be done efficiently by using Barvinok's algorithm [25- -27] (see also [28-30]).

The multiplicity formula itself has intrinsic interest beyond its application to algorithmics. One insight that is immediate from our result is the piecewise quasi-polynomial nature of the multiplicities $m_{\mu}^{\lambda}$ Corollary IV.3.

Let us now turn to the computation of the Kronecker coefficients $g_{\lambda, \mu, \nu}$, which arise in the decomposition of tensor products of irreducible representations of the symmetric group $S_{k}$ [31]:

$$
[\lambda] \otimes[\mu]=\bigoplus_{\nu} g_{\lambda, \mu, \nu}[\nu],
$$

where we denote by $[\lambda]$ the irreducible representation of $S_{k}$ labeled by the Young diagram $\lambda$ with $k$ boxes $\$ \mathrm{VI}$. Kronecker coefficients are notoriously difficult to study, and finding an appropriately strong combinatorial interpretation is one of the outstanding problems of classical representation theory. They appear naturally in geometric complexity theory, where their efficient computation has been subject to various conjectures [16], as well as in quantum information theory in the context of the marginal problem and coding theory [10, 11, 32-35].

Using Schur-Weyl duality, the Kronecker coefficients for Young diagrams with a bounded number of rows can be equivalently characterized in terms of a single subgroup restriction problem for compact connected Lie groups (§VI). Therefore, by Theorem I.2 they can also be computed efficiently:

Corollary I.4. For any fixed $d \in \mathbb{Z}_{>0}$, there exists a polynomial-time algorithm for computing the Kronecker coefficient $g_{\lambda, \mu, \nu}$ given as input Young diagrams $\lambda, \mu$ and $\nu$ with at most $d$ rows. That is, the algorithm runs in $O(\operatorname{poly}(\log k))$ where $k$ is the number of boxes of the Young diagrams.

Corollary I.5. Positivity of Kronecker coefficients for Young diagrams with a bounded number of rows can be decided in polynomial time.

By specializing our technique, we get a clean closed-form expression for the Kronecker coefficients (Proposition VI.1, which not only nicely illustrates its effectiveness, but also implies piecewise quasi-polynomiality for bounded height (a feature that has only been noticed in a special case [36]). Moreover, it is immediate from our formula that the problem of computing Kronecker coefficients with unbounded height is in GapP, as first proved in [19].

Similar conclusions can be drawn for the plethysm coefficients, which can also be formulated in terms of subgroup restriction problems [37]. Like the Kronecker coefficients, they play a fundamental role in geometric complexity theory [15. [38] and quantum information theory [11, 39].

In practice, our algorithms appear to be rather fast as long as the rank of the Lie group $G$ is not too large. In the case of Kronecker coefficients for Young diagrams with two rows, we can easily go up to $k=10^{8}$ boxes using commodity hardware. In contrast, all other software packages known to the authors cannot go beyond only a moderate number of boxes ( $k=10^{2}$ on the same hardware as used above). Moreover, by distributing the computation of weight multiplicities onto several processors, we have been able to compute Kronecker coefficients for Young diagrams with three rows and $k=10^{5}$ boxes in a couple of minutes 1 We hope that our algorithm will provide a useful tool in experimental mathematics, theoretical physics, and geometric complexity theory.

Our final result concerns the asymptotics of multiplicities in the general algebro-geometric setup of the geometric complexity theory approach to proving the $\mathbf{V P} \neq \mathbf{V N P}$ conjecture, an algebraic version of the $\mathbf{P} \neq \mathbf{N P}$ conjecture. Recall that, in a nutshell, this approach amounts to showing that for certain pairs of projective subvarieties $X$ and $Y$ one is not contained in the other; this would then imply complexity-theoretic lower bounds. Both the permanent vs. determinant problem, which is equivalent to the VP vs. VNP problem [40], as well as the complexity of matrix multiplication [41] can be formulated in this framework [13-15, 42]. More concretely, let us denote by $m_{H, X, k}(\mu)$ the multiplicity of the dual of an irreducible $H$ representation $V_{H, \mu}$ in the $k$-th graded part of the coordinate ring of $X$, and similarly for $Y$ (cf. \$VII for precise definitions). Then,

$$
X \subseteq Y \Rightarrow m_{H, X, k}(\mu) \leq m_{H, Y, k}(\mu)
$$

for all $\mu$ and $k \geq 0$. Therefore, the existence of $\mu$ and $k$ such that $m_{H, X, k}(\mu)>m_{H, Y, k}(\mu)$ proves that $X \nsubseteq Y$; such a pair $(\mu, k)$ is called an obstruction [14]. One can relax this implication further and instead compare the support of the multiplicity functions,

$$
X \subseteq Y \Rightarrow\left(m_{H, X, k}(\mu) \neq 0 \Rightarrow m_{H, Y, k}(\mu) \neq 0\right) .
$$

Since computing multiplicities in general coordinate rings is a difficult problem, it is natural to instead study their asymptotic behavior. Following an idea of Strassen [43], it has been proposed in [42] to consider the moment polytope,

$$
\Delta_{X}:=\overline{\bigcup_{k=1}^{\infty}\left\{\frac{\mu}{k}: m_{H, X, k}(\mu) \neq 0\right\}},
$$

which is a compact convex polytope that represents the asymptotic support of the stretching function. Moment polytopes do have a geometric interpretation, which should facilitate their computation [44]. Clearly,

$$
X \subseteq Y \Rightarrow \Delta_{X} \subseteq \Delta_{Y} .
$$

However, preliminary results suggest that the right-hand side moment polytope $\Delta_{Y}$ might be trivially large in the cases of interest [15, 42, 45, 46], and therefore insufficient for finding complexity-theoretic obstructions.

\footnotetext{
${ }^{1}$ A preliminary implementation of the algorithm is available upon request from the authors.
} 
It has therefore recently been suggested to study the asymptotic growth of multiplicities (e.g., [47, §2.2]). The natural object is the Duistermaat-Heckman measure, which is defined as the weak limit

$$
\mathrm{DH}_{X}:=\lim _{k \rightarrow \infty} \frac{1}{k^{d_{X}}} \sum_{\mu \in \Lambda_{H,+}^{*}} m_{H, X, k}(\mu) \delta_{\mu / k},
$$

where $d_{X} \in \mathbb{Z}_{>0}$ is the appropriate exponent such that $\mathrm{DH}_{X}$ is a non-zero finite measure [48]. The Duistermaat-Heckman measure has a continuous density function $f_{X}$ with respect to Lebesgue measure on the moment polytope; it is supported on the entire moment polytope (both statements follow from the main result of [48]). For well-behaved varieties, Duistermaat-Heckman measures have a geometric interpretation [49-55], which makes their computation potentially much more tractable [56-58] (this connection is however less clear in the singular cases relevant to geometric complexity theory). In this context, our main technical result is the following (see §III for the proof):

Theorem I.6. The exponent $d_{X}$ is equal to $\operatorname{dim} X-R_{X}$, where $R_{X}$ is the number of positive roots of $H$ that are not orthogonal to all points of the moment polytope $\Delta_{X}$.

The significance of Theorem I.6 is that the order of growth of the "smoothed" multiplicities, as captured by the Duistermaat-Heckman measures, does only depend on the dimension of the orbit closures and on their moment polytopes.

Now suppose that we are in the situation that $X$ and $Y$ cannot be separated by using moment polytopes, i.e., $\Delta_{X} \subseteq$ $\Delta_{Y}$. For the orbit closures $X$ and $Y$ that one tries to separate in geometric complexity theory, one can show that $\operatorname{dim} X<$ $\operatorname{dim} Y$ [15, 42]. Then, $X \subseteq Y$ would imply that $d_{X}<d_{Y}$ Corollary VII.4. But this means that we cannot deduce from (I.1) and (I.3) a criterion of the form

$$
X \subseteq Y \Rightarrow f_{X}(\mu) \leq f_{Y}(\mu) \quad(\forall \mu),
$$

since in order to take the weak limit we need to divide by different powers of $k$. Therefore, Duistermaat-Heckman measures do not directly give rise to new obstructions, indicating that a more refined understanding of the behavior of multiplicities in coordinate rings might be required.

\section{Preliminaries}

In this paper we will use basic notions of the theory of compact Lie groups [37, 59-61]. Let $G$ be a compact connected Lie group with Lie algebra $\mathfrak{g}$. We fix a maximal torus $T_{G} \subseteq G$ and denote by $\mathfrak{t}_{G}$ its Lie algebra, the corresponding Cartan subalgebra. We write $\Lambda_{G}=$ ker exp $\left.\right|_{t_{G}}$ for the integral lattice and $\Lambda_{G}^{*}$ for the weight lattice, which we can consider as a subset of $\mathfrak{t}_{G}^{*}$. The Weyl group $W_{G}$ acts on $\mathfrak{t}_{G}^{*}$ by reflections through the hyperplanes orthogonal to the roots. Let us choose a set of positive roots $R_{G,+} \subseteq \Lambda_{G}^{*}$. This determines a positive Weyl chamber $\mathfrak{t}_{G,+}^{*}$, as well as a basis of fundamental weights $\left\{\omega_{1}^{G}, \ldots, \omega_{r_{G}}^{G}\right\}$, where $r_{G}=\operatorname{dim} T_{G}$ is the rank of the Lie group, and the Weyl vector $\rho=\frac{1}{2} \sum_{\alpha \in R_{G,+}} \alpha$. The set of dominant weights $\Lambda_{G,+}^{*}$ is by definition the intersection of the weight lattice and the positive Weyl chamber.

The fundamental theorem of the representation theory of compact connected Lie groups is the fact that the irreducible (complex) representations of $G$ can be labeled by their highest weight $\lambda \in \Lambda_{G,+}^{*}$ [61]; for every element $\lambda \in \Lambda_{G,+}^{*}$ there exists a unique irreducible representation $V_{G, \lambda}$ with this highest weight. Given an arbitrary finite-dimensional (complex) $G$ representation $V$, we can always decompose it into irreducible sub-representations $V \cong \bigoplus_{\lambda \in \Lambda_{G,+}^{*}} m_{G, V}(\lambda) V_{G, \lambda}$. We shall call the function $m_{G, V}$ thus defined the highest weight multiplicity function.

If we restrict the representation to the maximal torus, we can similarly decompose into irreducible representations. Since $T_{G}$ is a compact Abelian group, we can always jointly diagonalize its action, and it follows that the irreducible representations are one-dimensional. The joint eigenvalues can be encoded as a weight $\beta \in \Lambda_{G}^{*}$, and we will denote the corresponding irreducible representation of $T_{G}$ by $\mathbb{C}_{\beta}$. The decomposition $V \cong \bigoplus_{\beta \in \Lambda_{G}^{*}} m_{T_{G}, V}(\beta) \mathbb{C}_{\beta}$ then defines the weight multiplicity function $m_{T_{G}, V}$. We also set $[k]=\{1, \ldots, k\}$, and write $f \sim g$ for the asymptotic equivalence $\lim _{k \rightarrow \infty} f(k) / g(k)=1$.

An equivalent way of encoding weight multiplicities is in terms of the (formal) character,

$$
\operatorname{ch} V=\sum_{\beta} m_{T_{G}, V}(\beta) e^{\beta},
$$

which can be understood as the generating function of $m_{T_{G}, V}$. Formally, $\operatorname{ch} V$ is an element of the group ring $\mathbb{Z}\left[\Lambda_{G}^{*}\right]$, which consists of (finite) linear combinations of basis elements $e^{\beta}$ subject to the relation $e^{\beta} e^{\beta^{\prime}}=e^{\beta+\beta^{\prime}}$. The character of an irreducible representation $V_{G, \lambda}$ is given by the Weyl character formula [61, p. 319],

$$
\operatorname{ch} V_{G, \lambda}=\frac{\sum_{w \in W_{G}} \operatorname{det}(w) e^{w(\lambda+\rho)}}{e^{\rho} \prod_{\alpha \in R_{G,+}}\left(1-e^{-\alpha}\right)} .
$$

Observe that we have

$$
\begin{aligned}
\frac{1}{\prod_{\alpha \in R_{G,+}}\left(1-e^{-\alpha}\right)} & =\prod_{\alpha \in R_{G,+}}\left(1+e^{-\alpha}+e^{-2 \alpha}+\ldots\right) \\
& =\sum_{\beta \in \Lambda_{G}^{*}} \phi_{R_{G,+}}(\beta) e^{-\beta},
\end{aligned}
$$

where $\phi_{R_{G,+}}$ is the Kostant partition function given by the formula

$$
\phi_{R_{G,+}}(\beta)=\#\left\{\left(x_{j}\right) \in \mathbb{Z}_{\geq 0}^{\left|R_{G,+}\right|}: \sum_{j} x_{j} \alpha_{j}=\beta\right\} .
$$

That is, $\phi_{R_{G,+}}$ counts the number of ways that a weight can be written as a sum of positive roots (this number is always finite since the positive roots span a proper cone). It follows directly from (II.1) and (III.2) and that

$$
\begin{aligned}
\operatorname{ch} V_{G, \lambda} & =\sum_{w \in W_{G}} \operatorname{det}(w) \sum_{\beta \in \Lambda_{G}^{*}} \phi_{R_{G,+}}(\beta) e^{w(\lambda+\rho)-\rho-\beta} \\
& =\sum_{\beta \in \Lambda_{G}^{*}} \sum_{w \in W_{G}} \operatorname{det}(w) \phi_{R_{G,+}}(w(\lambda+\rho)-\rho-\beta) e^{\beta} .
\end{aligned}
$$


In other words, the multiplicity of a weight $\beta$ in an irreducible representation $V_{G, \lambda}$ is given by the well-known Kostant multiplicity formula [20],

$$
m_{T_{G}, V_{G, \lambda}}(\beta)=\sum_{w \in W_{G}} \operatorname{det}(w) \phi_{R_{G,+}}(w(\lambda+\rho)-\rho-\beta) .
$$

For any fixed group $G$, the Kostant partition function can be evaluated efficiently by using Barvinok's algorithm [25], since it amounts to counting points in a convex polytope in an ambient space of fixed dimension. Therefore, weight multiplicities for fixed groups $G$ can be computed efficiently. This idea has been implemented by Cochet [21] to compute weight multiplicities for the classical Lie algebras (using the method presented in [30] instead of Barvinok's algorithm). We remark that the problem of computing weight multiplicities is of course the special case of Problem I.1 where $H$ is the maximal torus $T_{G} \subseteq G$.

\section{Weight Multiplicities as a Single Partition Function}

If $G$ is semisimple, we can find $s, t \in \mathbb{Z}_{\geq 0}$ and group homomorphisms $A: \mathbb{Z}^{s} \rightarrow \mathbb{Z}^{t}$ and $B: \Lambda_{G}^{*} \oplus \Lambda_{G}^{*} \rightarrow \mathbb{Z}^{t}$ such that

$$
m_{T_{G}, V_{G, \lambda}}(\beta)=\phi_{A}\left(B\left(\begin{array}{l}
\lambda \\
\beta
\end{array}\right)\right) \quad\left(\forall \lambda \in \Lambda_{G,+}^{*}, \beta \in \Lambda_{G}^{*}\right),
$$

where $\phi_{A}$ is the vector partition function defined by

$$
\phi_{A}(y)=\#\left\{x \in \mathbb{Z}_{\geq 0}^{s}: A x=y\right\} .
$$

Note that this improves over the Kostant multiplicity formula (II.4), where weight multiplicities are expressed as an alternating sum over vector partition functions. In particular, (II.5) is an evidently positive formula. It has been established by Billey, Guillemin, and Rassart for the Lie algebra $\mathfrak{s u}(d)$ [22], and was later extended to the general case by Bliem [23] by considering Littelmann patterns [62] instead of Gelfand-Tsetlin patterns [63].

The assumption of semisimplicity for (II.5) is not a restriction. Indeed, if $G$ is a general compact connected Lie group then its Lie algebra can always decomposed as

$$
\mathfrak{g}=[\mathfrak{g}, \mathfrak{g}] \oplus \mathfrak{z},
$$

where the commutator $[\mathfrak{g}, \mathfrak{g}]$ is the Lie algebra of a compact connected semisimple Lie group $G_{\mathrm{sS}}$, and where $\mathfrak{z}$ the Lie algebra of the center $Z(G)$ of $G$ [61, Corollary 4.25]. Let us choose a maximal torus $T_{G_{\mathrm{ss}}}$ of $G_{\mathrm{ss}}$ that is contained in $T_{G}$. Consider now an irreducible $G$-representation $V_{G, \lambda}$ with highest weight $\lambda$. By Schur's lemma, each element in $Z(G)$ acts by a scalar. Therefore, all weights $\beta$ that appear in the weight-space decomposition have the same restriction to $\mathfrak{z}$. It follows that

$$
m_{T_{G}, V_{G, \lambda}}(\beta)= \begin{cases}m_{T_{G_{\mathrm{ss}}}, V_{G_{\mathrm{ss}}, \lambda_{\mathrm{ss}}}}\left(\beta_{\mathrm{ss}}\right) & \text { if } \lambda_{z}=\beta_{z}, \\ 0 & \text { otherwise }\end{cases}
$$

where we write $\mu_{\mathrm{ss}}$ and $\mu_{z}$ for the restriction of a weight $\mu$ to the Cartan subalgebra of $[\mathfrak{g}, \mathfrak{g}]$ and to $\mathfrak{z}$, respectively. These multiplicities can therefore be evaluated by using (II.5).

\section{The Finite DifFEREnCE Formula}

Let $V$ be an arbitrary finite-dimensional representation of the compact, connected Lie group $G$. Clearly, we can compute the weight multiplicity function $m_{T_{G}, V}$ from the highest weight multiplicity function $m_{G, V}$ by using any of the classical formulas (II.1) and (II.4), or by evaluating the vector partition function (II.5) described in \$II. By "inverting" the Weyl character formula, the converse can also be achieved:

Proposition III.1. The highest weight and weight multiplicity function of a finite-dimensional $G$-representation $V$ are related by

$$
m_{G, V}=\left.\left(\prod_{\alpha \in R_{G,+}}-D_{\alpha}\right) m_{T_{G}, V}\right|_{\Lambda_{G,+}^{*}},
$$

where $\left(D_{\alpha} m\right)(\lambda)=m(\lambda+\alpha)-m(\lambda)$ is the finite-difference operator in direction $\alpha$. Note that any two of the operators $D_{\alpha}$ commute, so that their product is independent of the order of multiplication.

Proof: By linearity, it suffices to establish the lemma for a single irreducible representation $V=V_{G, \lambda}$ of highest weight $\lambda$. The Weyl character formula (II.1) can be rewritten in the form

$$
\prod_{\alpha>0}\left(1-e^{-\alpha}\right) \operatorname{ch} V_{G, \lambda}=\sum_{w \in W_{G}} \operatorname{det}(w) e^{w(\lambda+\rho)-\rho} .
$$

If we identify elements in $\mathbb{Z}\left[\Lambda_{G}^{*}\right]$ with functions on the weight lattice, applying finite-difference operators $D_{\alpha}$ corresponds to multiplication by $\left(e^{-\alpha}-1\right)$. Therefore, the left-hand side of (III.1) is identified with $\left(\prod_{\alpha \in R_{G,+}}-D_{\alpha}\right) m_{T_{G}, V_{G, \lambda}}$.

Now consider the right-hand side of (III.1). Since $\lambda+\rho$ is a strictly dominant weight, it is sent by any Weyl group element $w \neq 1$ to the interior of another Weyl chamber. That is, there exists a positive root $\alpha \in R_{G,+}$ such that $\langle\alpha, w(\lambda+\rho)\rangle<0$. In particular, $w(\lambda+\rho)-\rho$ is never dominant unless $w=1$. It follows that the restriction of $\left(\prod_{\alpha \in R_{G,+}}-D_{\alpha}\right) m_{T_{G}, V_{G, \lambda}}$ to $\Lambda_{G,+}^{*}$ is equal to the indicator function of $\{\lambda\}$, i.e., equal to the highest weight multiplicity function of $V_{G, \lambda}$.

The idea of using (II.1) for determining multiplicities of irreducible representations goes back at least to Steinberg [64], who proved a formula for the multiplicity $c_{\lambda, \mu}^{\nu}$ of an irreducible representation $V_{G, \nu}$ in the tensor product $V_{G, \lambda} \otimes$ $V_{G, \mu}$. These multiplicities $c_{\lambda, \mu}^{\nu}$ are called the LittlewoodRichardson coefficients for $G$. Steinberg's formula involves an alternating sum over the Kostant partition function (II.4); it can be evaluated efficiently as described by Cochet [21]. De Loera and McAllister give another method for computing Littlewood-Richardson coefficients [65], which applies Barvinok's algorithm to results by Berenstein and Zelevinsky [66]. Since the tensor products of irreducible $G$-representations are just the irreducible representations of $G \times G$, the problem of computing Littlewood-Richardson coefficients is again a special case of Problem I.1 The following consequence of the proof of Proposition III.1 will be convenient in the sequel: 
Corollary III.2. Write $\prod_{\alpha \in R_{G,+}}\left(1-e^{-\alpha}\right)=\sum_{\gamma \in \Gamma_{G}} c_{\gamma} e^{-\gamma}$ with $\Gamma_{G} \subseteq \Lambda_{G}^{*}$ finite and all $c_{\gamma} \neq 0$. Then,

$$
m_{G, V}(\lambda)=\sum_{\gamma \in \Gamma_{G}} c_{\gamma} m_{T_{G}, V}(\lambda+\gamma) .
$$

In particular, it is evident from Corollary III.2 that, for any fixed group $G$, the multiplicity of an irreducible representation in some representation $V$ can be computed efficiently from the weight multiplicities of $V$ by computing a finite linear combination.

\section{Multiplicities For the Subgroup Restriction PROBLEM}

Every $G$-representation $V$ can be considered as ("restricts to") a representation of $H$ by setting

$$
h \cdot v:=f(h) \cdot v \quad(\forall h \in H),
$$

and the subgroup restriction problem for $f$, as defined in Problem I.1, amounts to determining the multiplicity $m_{\mu}^{\lambda}$ of a given irreducible representation of $H$ in the restriction of a given irreducible representation of $G$. In this section we will derive a formula for these multiplicities (Theorem IV.2), which will be the main ingredient of the algorithm presented in $\S \mathrm{V}$ below. It will also follow from this formula that the $m_{\mu}^{\lambda}$ are given by a piecewise quasi-polynomial function 2 in $\lambda$ and $\mu$ (Corollary IV.3.

Let us choose the maximal torus $T_{H} \subseteq H$ in such a way that $f\left(T_{H}\right) \subseteq T_{G}$, and denote the corresponding Cartan subalgebra by $\mathfrak{t}_{H}$. Of course, this implies that the induced Lie algebra homomorphism Lie $(f)$ sends the Cartan subalgebra of $H$ in the one of $G$. Since $f$ is a group homomorphism, Lie $(f)$ restricts to a homomorphism between the integral lattices, $F: \Lambda_{H} \rightarrow \Lambda_{G}, X \mapsto \operatorname{Lie}(f) X$. The dual map between the weight lattices is given by

$$
F^{*}: \Lambda_{G}^{*} \rightarrow \Lambda_{H}^{*}, \quad \beta \mapsto \beta \circ F=\left.\beta \circ \operatorname{Lie}(f)\right|_{\Lambda_{H}} .
$$

The following is well-known and easily follows from the definitions:

Lemma IV.1. Let $V$ be a representation of $G$ and $v \in V a$ weight vector of weight $\beta \in \Lambda_{G}^{*}$. If we restrict the action to $H$ via (IV.1) then $v$ is a weight vector of weight $F^{*}(\beta) \in \Lambda_{H}^{*}$.

Let us also fix systems of positive roots $R_{H,+}$ for $H$. This in turn determines the set of dominant weights $\Lambda_{H,+}^{*}$ as well as a basis of fundamental weights $\left(\omega_{j}^{H}\right)$ as described in $\S \Pi$ Let us also set $r_{H}=\operatorname{dim} T_{H}$.

Our strategy for solving the subgroup restriction problem for $f$ then is the following: Given an irreducible representation $V_{G, \lambda}$ of $G$, we can determine its weight multiplicities with respect to the maximal torus $T_{G}$ by using any of the formulas presented in $\S$.II. We then obtain weight multiplicities for $T_{H}$ by restricting according to Lemma IV.1

\footnotetext{
${ }^{2}$ In the context of this paper, a quasi-polynomial function is a polynomial function with periodic coefficients; see p. 6for the precise definition. It should not to be confused with the notion of quasi-polynomial time complexity.
}

Finally, we reconstruct the multiplicity of an irreducible representation $V_{H, \mu}$ by using the finite-difference formula (Proposition III.1,Corollary III.2). If this procedure was translated directly into an algorithm, the runtime would be polynomial in the coefficients of $\lambda$ (with respect to the basis of fundamental weights), i.e., exponential in their bitlength, since the number of weights is of the order of the dimension of the irreducible representation $V_{G, \lambda}$, which according to the Weyl dimension formula is given by the polynomial $\prod_{\alpha \in R_{G,+}}\langle\alpha, \lambda+\rho\rangle /\langle\alpha, \rho\rangle$ (cf. the formula by Straumann [67]). We will now show that it is possible to combine the weight multiplicity formula (II.5) with the restriction map $F^{*}$ in a way that will later give rise to an algorithm that runs in polynomial time in the bitlength of the input:

Theorem IV.2. Let $f: H \rightarrow G$ be a homomorphism of compact connected Lie groups. Then we can find $s, s^{\prime}, u \in \mathbb{Z}_{\geq 0}$ and group homomorphisms $\mathcal{A}: \mathbb{Z}^{s+s^{\prime}} \rightarrow \mathbb{Z}^{u}$ and $\mathcal{B}: \Lambda_{G}^{*} \oplus$ $\Lambda_{H}^{*} \rightarrow \mathbb{Z}^{u}$ with the following property: For every irreducible representation $V_{G, \lambda}$ of $G$ and $V_{H, \mu}$ of $H$, the multiplicity $m_{\mu}^{\lambda}$ of the latter in the former is given by

$$
m_{\mu}^{\lambda}=\sum_{\gamma \in \Gamma_{H}} c_{\gamma} \#\left\{x \in \mathbb{Z}_{\geq 0}^{s} \oplus \mathbb{Z}^{s^{\prime}}: \mathcal{A} x=\mathcal{B}\left(\begin{array}{c}
\lambda \\
\mu+\gamma
\end{array}\right)\right\},
$$

where the (finite) set $\Gamma_{H}$ and the coefficients $\left(c_{\gamma}\right)$ are defined by $\prod_{\alpha \in R_{H,+}}\left(1-e^{-\alpha}\right)=\sum_{\gamma \in \Gamma_{H}} c_{\gamma} e^{-\gamma}$ and $c_{\gamma} \neq 0$. In fact, we can choose $s=O\left(r_{G}^{2}\right), s^{\prime} \leq r_{G}$ and $u=O\left(r_{G}^{2}\right)+r_{H}$.

Proof: By definition and Corollary III.2, we have $m_{\mu}^{\lambda}=$ $m_{H, V_{G, \lambda}}(\mu)=\sum_{\gamma \in \Gamma_{H}} c_{\gamma} m_{T_{H}, V_{G, \lambda}}(\mu+\gamma)$. In view of Lemma IV.1, the multiplicity of a $T_{H}$-weight $\delta \in \Lambda_{H}^{*}$ in the irreducible $G$-representation $V_{G, \lambda}$ is given by

$$
m_{T_{H}, V_{G, \lambda}}(\delta)=\sum_{\substack{\beta \in \Lambda_{G}^{*} \\ F^{*}(\beta)=\delta}} m_{T_{G}, V_{G, \lambda}}(\beta) .
$$

As in (II.7), let us now decompose the Lie-algebra $\mathfrak{g}=[\mathfrak{g}, \mathfrak{g}] \oplus$ $\mathfrak{z}$. Denote the Lie group corresponding to $[\mathfrak{g}, \mathfrak{g}]$ by $G_{\mathrm{ss}}$ and choose a maximal torus $T_{G_{s s}}$ which is contained in $T$. Using (II.8),

$$
\sum_{\substack{\beta \in \Lambda_{G}^{*} \\ F^{*}(\beta)=\delta}} m_{T_{G}, V_{G, \lambda}}(\beta)=\sum_{\substack{\beta_{\mathrm{ss}} \in \Lambda_{G_{\mathrm{ss}}}^{*} \\ C_{\mathrm{ss}} \beta_{\mathrm{ss}}+C_{z} \lambda_{z}=\delta}} m_{T_{G_{\mathrm{ss}}}, V_{G_{\mathrm{ss}}, \lambda_{\mathrm{ss}}}}\left(\beta_{\mathrm{ss}}\right),
$$

where we have decomposed $F^{*}$ as a sum of two homomorphisms $C_{\mathrm{ss}}: \Lambda_{G_{\mathrm{ss}}}^{*} \rightarrow \Lambda_{H}^{*}$ and $C_{z}: \Lambda_{Z(G)}^{*} \rightarrow \Lambda_{H}^{*}$.

Let us now choose group homomorphisms $A: \mathbb{Z}^{s} \rightarrow \mathbb{Z}^{t}$ and $B=B_{1} \oplus B_{2}: \Lambda_{G_{s \mathrm{~s}}}^{*} \oplus \Lambda_{G_{\mathrm{ss}}}^{*} \rightarrow \mathbb{Z}^{t}$ such that (II.5) holds for the weight multiplicities for $G_{\mathrm{ss}}$. For this, $s$ and $t$ can be taken 
of order $O\left(r_{G}^{2}\right)$ [23, Proposition 19]. Then,

$$
\begin{aligned}
& \sum_{\beta_{\mathrm{ss}} \in \Lambda_{G_{\mathrm{ss}}}^{*}} m_{T_{G_{\mathrm{ss}}}, V_{G_{\mathrm{ss}}, \lambda_{\mathrm{ss}}}}\left(\beta_{\mathrm{ss}}\right) \\
& C_{\mathrm{ss}} \beta_{\mathrm{ss}}+C_{z} \lambda_{z}=\delta \\
& =\sum_{\beta_{\mathrm{ss}} \in \Lambda_{G_{\mathrm{ss}}}^{*}} \#\left\{x \in \mathbb{Z}_{\geq 0}^{s}: A x=B\left(\begin{array}{c}
\lambda_{\mathrm{ss}} \\
\beta_{\mathrm{ss}}
\end{array}\right)\right\} \\
& C_{\mathrm{ss}} \beta_{\mathrm{ss}}+C_{z} \lambda_{z}=\delta \\
& =\#\left\{\left(x, \beta_{\mathrm{ss}}\right):\left(\begin{array}{cc}
A & -B_{2} \\
0 & C_{\mathrm{ss}}
\end{array}\right)\left(\begin{array}{c}
x \\
\beta_{\mathrm{ss}}
\end{array}\right)=\left(\begin{array}{c}
B_{1} \lambda_{\mathrm{ss}} \\
-C_{z} \lambda_{z}+\delta
\end{array}\right)\right\} \\
& =\#\{\left(x, \beta_{\mathrm{ss}}\right): \underbrace{\left(\begin{array}{cc}
A & -B_{2} \\
0 & C_{\mathrm{ss}}
\end{array}\right)}_{=: \mathcal{A}}\left(\begin{array}{c}
x \\
\beta_{\mathrm{ss}}
\end{array}\right)=\underbrace{\left(\begin{array}{ccc}
B_{1} & 0 & 0 \\
0 & -C_{z} & \mathbf{1}
\end{array}\right)}_{=: \mathcal{B}}\left(\begin{array}{c}
\lambda_{\mathrm{ss}} \\
\lambda_{z} \\
\delta
\end{array}\right)\} .
\end{aligned}
$$

After choosing a basis of the lattice $\Lambda_{G_{\mathrm{ss}}}^{*}$ we arrive at the asserted formula (with $s^{\prime}=\operatorname{dim} T_{G_{\mathrm{ss}}}$ and $u=t+r_{H}$ ).

We stress that the proof of Theorem IV.2 is constructive: The maps $\mathcal{A}$ and $\mathcal{B}$, whose existence is asserted by the theorem, are defined in IV.3 in terms of $A$ and $B$, whose construction is described explicitly in [22, Proof of Theorem 2.1] (for the case of $\mathfrak{g}=\mathfrak{s u}(d)$ ) and in [23, §4] (for the general case). See $\$ \mathrm{VI}$ for an illustration in the context of the Kronecker coefficients.

If one uses the Kostant multiplicity formula (II.4) instead of (II.5) in the proof of Theorem IV.2 then one arrives at a similar formula for the multiplicities $m_{\mu}^{\lambda}$ involving an additional alternating sum over the Weyl group of $G$. After completion of this work, we have learned of [49. Lemma 3.1] which is derived in this spirit.

\section{Piecewise Quasi-Polynomiality}

Let us use the fundamental weight bases fixed above to identify $\Lambda_{G}^{*} \cong \mathbb{Z}^{r_{G}}$ and $\Lambda_{H}^{*} \cong \mathbb{Z}^{r_{H}}$. The group homomorphisms $\mathcal{A}$ and $\mathcal{B}$ correspond to matrices with integer entries, which we shall denote by the same symbols. Observe that the formula in Theorem IV.2 in essence amounts to counting the number $n(y):=\#\left(\Delta_{\mathcal{A}, \mathcal{B}}(y) \cap \mathbb{Z}^{s+s^{\prime}}\right)$ of integral points in certain rational convex polytopes of the form

$$
\Delta_{\mathcal{A}, \mathcal{B}}(y):=\left\{x \in \mathbb{R}^{s+s^{\prime}}: x_{1}, \ldots, x_{s} \geq 0, \mathcal{A} x=\mathcal{B} y\right\}
$$

parametrized by $y \in \mathbb{Z}^{r_{G}+r_{H}}$. Explicitly,

$$
m_{\mu}^{\lambda}=\sum_{\gamma \in \Gamma_{H}} c_{\gamma} n(\lambda, \mu+\gamma) .
$$

It is well-known that $n(y)$ is a piecewise quasi-polynomial function in $y$ [68]. That is, there exists a decomposition of $\mathbb{Z}^{r_{G}+r_{H}}$ into polyhedral chambers such that on each chamber $C$ the function $n(y)$ is given by a single quasi-polynomial, i.e., there exists a sublattice $L \subseteq \mathbb{Z}^{r_{G}+r_{H}}$ of finite index and polynomials $\left(p_{z}\right)$ with rational coefficients, labeled by the finitely many points $z \in \mathbb{Z}^{r_{G}+r_{H}} / L$, such that $n(y)=p_{[y]}(y)$ for all $y \in \mathbb{Z}^{r_{G}+r_{H}}$ (cf. [69, §2.2]). We record the following immediate consequence:

Corollary IV.3. For any fixed group homomorphism $f: H \rightarrow$ $G$, the multiplicities $m_{\mu}^{\lambda}$ are given by a piecewise quasipolynomial function in $\lambda$ and $\mu$.
In particular, this implies that the stretching function $k \mapsto$ $m_{k \mu}^{k \lambda}$ is a quasi-polynomial function for large $k$. This is in fact true for all $k$, as has been observed in [16] (cf. [53] for more general quasi-polynomiality results on convex cones, and also [70] for further discussion).

\section{Polynomial-Time Algorithm for the Subgroup RESTRICTION PROBLEM}

In this section we will formulate our algorithm for the subgroup restriction problem, Problem I.1 Recall that, by (IV.5), the computation of the multiplicities $m_{\mu}^{\lambda}$ effectively reduces to counting the number of integral points in certain rational convex polytopes of the form (IV.4). We shall suppose that the highest weights $\lambda$ and $\mu$, which are the input to our algorithm, are given in terms of their coordinates with respect to the fundamental weight bases fixed in \$IV Clearly, for each of the finitely many $\gamma \in \Gamma_{H}$, the description of the polytope $\Delta_{\mathcal{A}, \mathcal{B}}(\lambda, \mu+\gamma)$ (say, in terms of linear inequalities) is of polynomial size in the bitlength of the input. It follows that Barvinok's algorithm can be used to compute the number of integral points in each of these polytopes in polynomial time [25] (see also [26, 27]). This gives rise to the following polynomial-time algorithm for Problem I.1 thereby establishing Theorem I.2.

Algorithm V.1. Let $f: H \rightarrow G$ be a homomorphism of compact connected Lie groups. Given as input two highest weights $\lambda \in \Lambda_{G}^{*} \cong \mathbb{Z}^{r_{G}}$ and $\mu \in \Lambda_{H}^{*} \cong \mathbb{Z}^{r_{H}}$, encoded as bitstrings containing their coordinates with respect to the fundamental weight bases fixed above, the following algorithm computes the multiplicity $m_{\mu}^{\lambda}$ in polynomial time in the bitlength of the input:

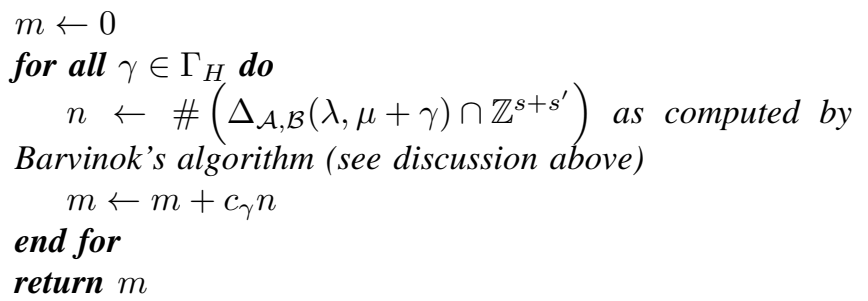

Here, $\Delta_{\mathcal{A}, \mathcal{B}}(y)$ denotes the rational convex polytope defined in (IV.4), and the finite index set $\Gamma_{H} \subseteq \Lambda_{H}^{*}$ as well as the coefficients $\left(c_{\gamma}\right)$ are defined in the statement of Theorem IV.2

There are at least two software packages which have implemented Barvinok's algorithm, namely LATTE [71] and BARVINOK [69, 72]. In \$I we have reported on the performance of our implementation of Algorithm V.1 for computing Kronecker coefficients using the latter package.

Remark V.2. The existence of a polynomial-time algorithm for Problem I.1 in fact already follows abstractly from Corollary IV.3. since in order to compute $m_{\mu}^{\lambda}$ we merely have to evaluate a fixed piecewise quasi-polynomial function. This piecewise quasi-polynomial can be computed algorithmically by using a variant of Barvinok's algorithm which is also implemented in the BARVINOK package; see [69. Proposition 2] and also [27, (5.3.1)]. 


\section{KRONECKER COEFFICIENTS}

As explained in the introduction, the Kronecker coefficients play an important role in geometric complexity theory and quantum information theory. In this section, we will describe precisely how they can be computed using our methods.

Let us recall the language of Young diagrams which is commonly used in this context [31]. A Young diagram with $r$ rows and $k$ boxes is given by an ordered list of integers $\lambda_{1} \geq \ldots \geq \lambda_{r}>0$ with $\sum_{i} \lambda_{i}=k$. It can be visualized as an arrangement of $k$ boxes in $r$ rows with $\lambda_{j}$ boxes in the $j$-th row. We set $\lambda_{j}=0$ for all $j>r$. We will now consider the unitary group $\mathrm{U}(d)$, which consists of the unitary $d \times d$ matrices. Let us fix a system of positive roots and denote the corresponding basis of fundamental weights by $\left(\omega_{j}\right)$. To each Young diagram $\lambda$ with at most $d$ rows we associate the irreducible representation of $\mathrm{U}(d)$ with highest weight equal to $\sum_{j=1}^{d}\left(\lambda_{j}-\lambda_{j+1}\right) \omega_{j}$. Every polynomial irreducible representation of $\mathrm{U}(d)$ arises in this way. By a slight abuse of notation, we identify Young diagrams with the corresponding highest weights. More generally, we can associate to every integer vector $\beta \in \mathbb{Z}^{d}$ the weight $\sum_{j=1}^{d}\left(\beta_{j}-\beta_{j+1}\right) \omega_{j}$, where we set $\beta_{d+1}=0$. This defines a bijection between $\mathbb{Z}^{d}$ and the weight lattice $\Lambda_{\mathrm{U}(d)}^{*}$ of $\mathrm{U}(d)$. In particular, the positive roots fixed above correspond to the integer vectors of the form $(\ldots, 0,1,0, \ldots, 0,-1,0, \ldots)$.

The Kronecker coefficient $g_{\lambda, \mu, \nu}$ associated with triples of Young diagrams $\lambda, \mu$ and $\nu$ with $k$ boxes each and at most $a$, $b$ and $c$ rows, respectively, can then be defined in terms of the following subgroup restriction problem of compact, connected Lie groups: Let $H=\mathrm{U}(a) \times \mathrm{U}(b) \times \mathrm{U}(c)$ and $G=\mathrm{U}(a b c)$ and consider the homomorphism $f: H \rightarrow G$ given by sending a triple of unitaries $(U, V, W)$ to their tensor product $U \otimes$ $V \otimes W$. The Kronecker coefficient $g_{\lambda, \mu, \nu}$ is then given by the multiplicity of the irreducible $H$-representation $V_{H,(\lambda, \mu, \nu)}=$ $V_{\mathrm{U}(a), \lambda} \otimes V_{\mathrm{U}(b), \mu} \otimes V_{\mathrm{U}(c), \nu}$ in the restriction of the symmetric power $\operatorname{Sym}^{k}\left(\mathbb{C}^{a b c}\right)$, which is the irreducible $G$-representation labeled by the Young diagram $(k)$ consisting of a single row with $k$ boxes. That is,

$$
g_{\lambda, \mu, \nu}=m_{\lambda, \mu, \nu}^{(k)}(f)
$$

This definition in fact does not depend on the concrete values chosen for $a, b$ and $c$, as can be seen by rephrasing it in terms of the representation theory of the symmetric group $S_{k}$ [15, $\S 8$ ] (but of course $a, b$ and $c$ have to be chosen at least as large as the number of rows of the Young diagrams). Moreover, it is evident that the Kronecker coefficients are symmetric in the variables $\lambda, \mu$, and $\nu$.

It follows that, for any fixed choice of $a, b$ and $c$, Algorithm V.1 can be used to compute the Kronecker coefficient (VI.1) given Young diagrams with at most $a, b$ and $c$ rows, respectively, in polynomial time in the input size, or equivalently in time $O(\operatorname{poly}(\log k))$, where $k$ is the number of boxes of the Young diagrams. This establishes Corollary I.4 Let us again stress that the problem of computing Kronecker coefficients is known to be \#P-hard in general [19]; hence we do not expect that there exists a polynomial-time algorithm without any assumption on the number of rows of the Young diagrams.

When computing Kronecker coefficients using the above method, we are only interested in the representation $V_{\mathrm{U}(a b c),(k)}=\operatorname{Sym}^{k}\left(\mathbb{C}^{a b c}\right)$, not in arbitrary irreducible representations of $\mathrm{U}(a b c)$. By specializing the construction described in Theorem IV.2 to this one-parameter family of representations, we obtain the following result:

Proposition VI.1. The multiplicity of a weight $\delta=$ $\left(\delta^{A}, \delta^{B}, \delta^{C}\right) \in \mathbb{Z}^{a} \oplus \mathbb{Z}^{b} \oplus \mathbb{Z}^{c} \cong \Lambda_{H}^{*}$ (we use the identifications fixed at the beginning of $\$ \mathrm{SVT}$ in the irreducible $G$ representation $\operatorname{Sym}^{k}\left(\mathbb{C}^{a b c}\right)$ is equal to the number of integral points in the rational convex polytope

$$
\begin{aligned}
& \Delta(k, \delta)=\left\{\left(x_{l, m, n}\right) \in \mathbb{R}_{\geq 0}^{a b c}: \sum_{l, m, n} x_{l, m, n}=k,\right. \\
& \left.\sum_{m, n} x_{l, m, n}=\delta_{l}^{A}, \sum_{l, n} x_{l, m, n}=\delta_{m}^{B}, \sum_{l, m} x_{l, m, n}=\delta_{n}^{C}\right\} .
\end{aligned}
$$

It follows that the Kronecker coefficient for Young diagrams $\lambda, \mu, \nu$ with $k$ boxes and at most $a, b$ and $c$ rows, respectively, is given by the formula

$$
g_{\lambda, \mu, \nu}=\sum_{\gamma \in \Gamma_{H}} c_{\gamma} \#\left(\Delta(k,(\lambda, \mu, \nu)+\gamma) \cap \mathbb{Z}^{a b c}\right),
$$

where $\Gamma_{H}$ and $\left(c_{\gamma}\right)$ are defined as in the statement of Corollary III.2.

Proof: It is well-known that the weight spaces for the action of $\mathrm{U}(d)$ on $\operatorname{Sym}^{k}\left(\mathbb{C}^{d}\right)$ are all one-dimensional and that the set of weights corresponds to the integer vectors in the standard simplex rescaled by $k$ [31]. In our case, $d=a b c$, so that the weights are just the integral points of the polytope

$$
\left\{x=\left(x_{l, m, n}\right)_{l \in[a], m \in[b], n \in[c]} \in \mathbb{R}_{\geq 0}^{a b c}: \sum_{l, m, n} x_{l, m, n}=k\right\} .
$$

Moreover, the dual map $F^{*}: \Lambda_{\mathrm{U}(a b c)}^{*} \rightarrow \Lambda_{\mathrm{U}(a) \times \mathrm{U}(b) \times \mathrm{U}(c)}^{*}$ as defined in (IV.2) is given by

$$
\begin{cases}\mathbb{Z}^{a b c} & \rightarrow \mathbb{Z}^{a} \oplus \mathbb{Z}^{b} \oplus \mathbb{Z}^{c} \\ \left(x_{l, m, n}\right) & \mapsto\left(\sum_{m, n} x_{l, m, n}, \sum_{l, n} x_{l, m, n}, \sum_{l, m} x_{l, m, n}\right) .\end{cases}
$$

We conclude that the multiplicity of a weight $\delta=\left(\delta^{A}, \delta^{B}, \delta^{C}\right)$ for $\mathrm{U}(a) \times \mathrm{U}(b) \times \mathrm{U}(c)$ is given by the number of integral points in the polytope $\Delta(k, \delta)$ described above.

Just as for our main algorithm, Proposition VI.1 gives rise to a polynomial-time algorithm for computing Kronecker coefficients with a bounded number of rows. This second algorithm runs faster than the generic one presented earlier, since the ambient space $\mathbb{R}^{a b c}$ has a smaller dimension than what we would get from the construction described in the proof of Theorem IV.2 We remark that the time complexity for unbounded $a, b$ and $c$ can be deduced from [27]. 


\section{ASYMPTOTICS}

In this section we will prove our result on the generic order of growth of multiplicities in the coordinate ring of a projective variety (Theorem I.6).

We will work in the following general setup: Let $V$ be a finite-dimensional rational representation of $H$, and suppose that $X$ is an $H$-stable closed subvariety of the associated projective space $\mathbb{P}(V)$. The homogeneous coordinate ring $\mathbb{C}[X]$ is graded, and we can decompose each part into its irreducible components,

$$
\mathbb{C}[X]=\bigoplus_{k=0}^{\infty} \mathbb{C}[X]_{k}=\bigoplus_{k=0}^{\infty} \bigoplus_{\mu} m_{H, X, k}(\mu) V_{H, \mu}^{*},
$$

where, following the usual conventions, we have decomposed with respect to the dual representations $V_{H, \mu}^{*}$. The stretching function is then by definition $k \mapsto m_{H, X, k}(k \mu)$. We stress that in contrast to [16], where it was assumed that $X$ has at most rational singularities, we do not even require that $X$ is a normal variety [73]. This is highly relevant for geometric complexity theory, since it was recently shown in [74] and [42] that the studied varieties (the orbit closures of the determinant and permanent on the one hand, and of the matrix multiplication tensor and the unit tensor on the other hand) are in fact never normal except in trivial situations.

Remark VII.1. The subgroup restriction problem for a rational group homomorphism $f: H \rightarrow G$ can be realized in the above setup: Indeed, for any highest weight $\lambda \in \Lambda_{G,+}^{*}$ consider $X=\mathcal{O}_{G, \lambda}$, the coadjoint orbit through $\lambda$, with the induced action of $H$. This variety can be canonically embedded into projective space as the orbit of the highest weight vector in $\mathbb{P}\left(V_{G, \lambda}\right)$, and it is a consequence of the Borel-Weil theorem that $\mathbb{C}\left[\mathcal{O}_{G, \lambda}\right]=\bigoplus_{k=0}^{\infty} V_{G, k \lambda}^{*}$. By comparing with VII.1) it follows that $m_{H, \mathcal{O}_{G, \lambda}, k}(\mu)=m_{\mu}^{k \lambda}$. In particular, the above definition of the stretching function, $k \mapsto m_{H, \mathcal{O}_{G, \lambda}, k}(k \mu)$, coincides with our previous usage, $k \mapsto m_{k \mu}^{k \lambda}$.

Proof of Theorem I.6. By the Hilbert-Serre theorem, the function $k \mapsto \operatorname{dim} \mathbb{C}[X]_{k}$ is a polynomial of degree $\operatorname{dim} X$ for large $k$ [73, Theorem I.7.5]. Hence there exists a constant $A>0$ such that

$$
\begin{aligned}
& A k^{\operatorname{dim} X} \sim \operatorname{dim} \mathbb{C}[X]_{k} \\
= & \sum_{\mu \in \Lambda_{H,+}^{*}} m_{H, X, k}(\mu) \operatorname{dim} V_{\mu}=\sum_{\mu \in \Delta_{X} \cap \frac{1}{k} \Lambda_{H,+}^{*}} m_{H, X, k}(k \mu) \operatorname{dim} V_{k \mu},
\end{aligned}
$$

where for the last equality we have used the definition of the moment polytope $\Delta_{X}$. By the Weyl dimension formula, we have

$$
\begin{gathered}
\operatorname{dim} V_{k \mu}=\prod_{\alpha \in R_{H,+}} \frac{\langle\alpha, k \mu+\rho\rangle}{\langle\alpha, \rho\rangle} \\
=\left(\prod_{\substack{\alpha \in R_{H},+\alpha \not \lambda \Delta_{X}}} \frac{\langle\alpha, \mu\rangle}{\langle\alpha, \rho\rangle}\right) k^{R_{X}}+O\left(k^{R_{X}-1}\right)
\end{gathered}
$$

for the representations that occur in $\mathbb{C}[X]$. The coefficient $P(\mu)=\prod_{\alpha \in R_{H,+}, \alpha \not \Delta_{X}}\langle\alpha, \mu\rangle /\langle\alpha, \rho\rangle$ is a polynomial function in $\mu$. Since $\Delta_{X}$ is compact, we can therefore find a constant $C>0$ such that

$$
\operatorname{dim} V_{k \mu} \leq C k^{R_{X}} \quad\left(\forall k, \mu \in \Delta_{X} \cap \frac{1}{k} \Lambda_{H,+}^{*}\right) .
$$

It follows that

$$
\begin{aligned}
& \sum_{\mu \in \Delta_{X} \cap \frac{1}{k} \Lambda_{H,+}^{*}, X, k} m_{H, d}(k \mu) \operatorname{dim} V_{k \mu} \\
\leq & C k^{R_{X}} \sum_{\mu \in \Delta_{X} \cap \frac{1}{k} \Lambda_{H,+}^{*}} m_{H, X, k}(k \mu) \sim C k^{R_{X}+d_{X}} \int d \mathrm{DH}_{X},
\end{aligned}
$$

so that $\operatorname{dim} X \leq R_{X}+d_{X}$.

On the other hand, since $\mathrm{DH}_{X}$ is Lebesgue-absolutely continuous, the boundary of the moment polytope does not carry any measure. We can therefore find a compact set $K$ contained in the (relative) interior of the moment polytope which has positive measure with respect to $\mathrm{DH}_{X}$. Note that $P(\mu)$ is positive for all $\mu$ contained in the interior of the moment polytope (indeed, for all positive roots $\alpha$ with $\alpha \not \perp \Delta_{X}$ there exists $\nu \in \Delta_{X}$ such that $\langle\alpha, \nu\rangle>0$; since we can always write $\mu$ as a proper convex combination of $\nu$ and some other point $\nu^{\prime} \in \Delta_{X}$, it follows that $\langle\alpha, \mu\rangle>0$ ). This implies that on the compact set $K$ we can bound $P(\mu)$ from below by a positive constant. Thus there exists a constant $D>0$ (depending on $K)$ such that

$$
\operatorname{dim} V_{k \mu} \geq D k^{R_{X}} \quad\left(\forall \mu \in K \cap \frac{1}{k} \Lambda_{H,+}^{*}\right) .
$$

Consequently,

$$
\begin{aligned}
& \sum_{\mu \Delta_{X} \cap \frac{1}{k} \Lambda_{H,+}^{*}} m_{H, X, k}(k \mu) \operatorname{dim} V_{k \mu} \geq \sum_{\mu \in K \cap \frac{1}{k} \Lambda_{H,+}^{*}} m_{H, X, k}(k \mu) \operatorname{dim} V_{k \mu} \\
\geq & D k^{R_{X}} \sum_{\mu \in K \cap \frac{1}{k} \Lambda_{H,+}^{*}} m_{H, X, k}(k \mu) \sim D k^{R_{X}+d_{X}} \int_{K} d \mathrm{DH}_{X} .
\end{aligned}
$$

We conclude that also $\operatorname{dim} X \geq R_{X}+d_{X}$, hence we have equality.

Let us now elaborate on the argument presented at the end of the introduction, where we showed that Duistermaat-Heckman measures do not directly give rise to new complexity-theoretic obstructions. For this, we consider a pair of projective subvarieties $X$ and $Y$ with $\operatorname{dim} X<\operatorname{dim} Y$, as is the case for the orbit closures of relevance to GCT. Let us assume that $\Delta_{X} \subseteq \Delta_{Y}$, so that the moment polytopes alone do not already give rise to an obstruction. Clearly, this implies that $R_{X} \leq R_{Y}$.

Lemma VII.2. Let $\Delta_{X} \subseteq \Delta_{Y}$ and $R_{X}<R_{Y}$. Then, $\operatorname{dim} \Delta_{X}<\operatorname{dim} \Delta_{Y}$.

Proof: Note that we have

$$
\operatorname{dim} \Delta_{X}=\operatorname{dim} \text { aff } \Delta_{X} \leq \operatorname{dim} \text { aff } \Delta_{Y}=\operatorname{dim} \Delta_{Y},
$$

with equality if and only if the two affine hulls aff $\Delta_{X} \subseteq$ aff $\Delta_{Y}$ are equal. 
Now by assumption there exists a positive root $\alpha \in R_{H,+}$ that is orthogonal to all points in $\Delta_{X}$ (i.e., for all $p \in \Delta_{X}$, $\alpha \perp p$ ), but not to all points in $\Delta_{Y}$. It follows that $\alpha$ is also orthogonal to all points in the affine hull of $\Delta_{X}$, but not to all points in the affine hull of $\Delta_{Y}$. Therefore, we have aff $\Delta_{X} \subsetneq$ aff $\Delta_{Y}$

Lemma VII.3. Let $\operatorname{dim} \Delta_{X}<\operatorname{dim} \Delta_{Y}$. Then, $X \subseteq Y$ implies $d_{X}<d_{Y}$.

Proof: If $X \subseteq Y$ then it is immediate from (I.1) and (I.3) that $d_{X} \leq d_{Y}$. Let us suppose for a moment that in fact $d_{X}=d_{Y}$. Then it follows from (I.1) that

$$
\int_{\Delta_{X}} d \mathrm{DH}_{X}(\mu) g(\mu) \leq \int_{\Delta_{Y}} d \mathrm{DH}_{Y}\left(\mu^{\prime}\right) g\left(\mu^{\prime}\right)
$$

for any test function $g$. In particular, this inequality would hold for $g$ the indicator function of $\Delta_{X}$. But this is clearly impossible, since $\mathrm{DH}_{Y}$ is absolutely continuous with respect to Lebesgue measure on $\Delta_{Y}$, for which $\Delta_{X}$ is a set of measure zero.

Corollary VII.4. Let $\operatorname{dim} X<\operatorname{dim} Y$. Then, $X \subseteq Y$ implies $d_{X}<d_{Y}$.

Proof: Clearly, $X \subseteq Y$ implies that $\Delta_{X} \subseteq \Delta_{Y}$ and $R_{X} \leq R_{Y}$. If $R_{X}=R_{Y}$ then the assertion follows directly from Theorem I.6, since

$$
d_{X}=\operatorname{dim} X-R_{X}<\operatorname{dim} Y-R_{Y}=d_{Y} .
$$

Otherwise, if $R_{X}<R_{Y}$, it follows from combining Lemma VII.2 and Lemma VII.3

As described in the introduction, the upshot of the above is that we cannot directly deduce from (I.1) a new criterion for obstructions based on the Duistermaat-Heckman measure that goes beyond what is provided by the moment polytope.

\section{ACKNOWLEDGEMENTS}

We would like to thank Aravind Asok, Emmanuel Briand, Peter Bürgisser, David Gross, Christian Ikenmeyer, Stavros Kousidis, Graeme Mitchison, Mercedes Rosas, Volkher Scholz, and Michèle Vergne for helpful discussions.

This work is supported by the Swiss National Science Foundation (grant PP00P2-128455 and 200021_138071), the German Science Foundation (grants $\mathrm{CH}$ 843/1-1 and $\mathrm{CH}$ 843/2-1), and the National Center of Competence in Research 'Quantum Science and Technology'.

\section{REFERENCES}

[1] H. Weyl, The Theory of Groups and Quantum Mechanics. Dover, 1950.

[2] E. P. Wigner, Group Theory and its Application to the Quantum Mechanics of Atomic Spectra. Academic Press Inc., 1959, translated by J. J. Griffin.

[3] _ , "Reduction of Direct Products and Restriction of Representations to Subgroups: The Everyday Tasks of the Quantum Theorists," SIAM J. Appl. Math., vol. 25, pp. 169-185, 1973.

[4] Y. Nee'man, "Derivation of strong interactions from a gauge invariance," Nuclear Phys., vol. 26, pp. 222-229, 1961.

[5] M. Gell-Mann, "Synchrotron Laboratory Report (CTSL-20)," California Institute of Technology, Tech. Rep., 1961.
[6] - "Symmetries of baryons and mesons," Phys. Rev., vol. 125, pp. $1067-1084,1962$.

[7] W. Fulton, "Eigenvalues, invariant factors, highest weights, and Schubert calculus," Bull. Amer. Math. Soc., vol. 37, pp. 209-249, 2000.

[8] A. Knutson and T. Tao, "The honeycomb model of $\mathrm{GL}_{n}(\mathbb{C})$ tensor products I: Proof of the saturation conjecture," J. Amer. Math. Soc., vol. 12, pp. 1055-1090, 1999.

[9] M. Keyl and R. F. Werner, "Estimating the spectrum of a density operator," Phys. Rev. A, vol. 64, p. 052311, 2001

[10] M. Christandl and G. Mitchison, "The Spectra of Quantum States and the Kronecker Coefficients of the Symmetric Group," Commun. Math. Phys., vol. 261, pp. 789-797, 2006.

[11] A. Klyachko, "Quantum marginal problem and $N$-representability," $J$. Phys.: Conf. Ser., vol. 36, pp. 72-86, 2006.

[12] D. Bacon, I. L. Chuang, and A. W. Harrow, "The Quantum Schur Transform: I. Efficient Qudit Circuits," in Proc. 18th SODA, 2007, pp. $1235-1244$

[13] K. D. Mulmuley and M. Sohoni, "Geometric complexity theory I: An approach to the P vs. NP and related problems," SIAM J. Comput., vol. 31, pp. 496-526, 2001

[14] — " "Geometric complexity theory II: Towards explicit obstructions for embeddings among class varieties," SIAM J. Comput., vol. 38, pp. 1175-1206, 2008.

[15] P. Bürgisser, J. M. Landsberg, L. Manivel, and J. Weyman, "An Overview of Mathematical Issues arising in the Geometric Complexity Theory approach to $V P \neq V N P$," SIAM J. Comput., vol. 40, pp. 1179-1209, 2011.

[16] K. D. Mulmuley, "Geometric complexity theory VI: the flip via saturated and positive integer programming in representation theory and algebraic geometry," Computer Science Department, The University of Chicago, Technical Report, 2007.

[17] K. D. Mulmuley and M. Sohoni, "Geometric complexity theory III: On deciding positivity of Littlewood-Richardson coefficients," arXiv:cs/0501076, 2005.

[18] H. Narayanan, "On the complexity of computing Kostka numbers and Littlewood-Richardson coefficients," J. Alg. Combinat., vol. 24, pp. 347$354,2006$.

[19] P. Bürgisser and C. Ikenmeyer, "The complexity of computing Kronecker coefficients," in Proc. 20th FPSAC. DMTCS, 2008, pp. 357-368.

[20] B. Kostant, "A formula for the multiplicity of a weight," Transact. Amer Math. Soc., vol. 93, pp. 53-73, 1959.

[21] C. Cochet, "Vector partition function and representation theory," in Proc. 17th FPSAC. DMTCS, 2005.

[22] S. Billey, V. Guillemin, and E. Rassart, "A vector partition function for the multiplicities of $\mathfrak{s l}_{k}(\mathbf{C})$," J. Algebra, vol. 278, pp. 251-293, 2004.

[23] T. Bliem, "On weight multiplicities of complex simple Lie algebras," PhD Thesis, Universität zu Köln, 2008.

[24] — "Chopped and sliced cones and representations of Kac-Moody algebras," J. Pure Appl. Algebra, vol. 214, pp. 1152-1164, 2010.

[25] A. Barvinok, "A Polynomial Time Algorithm for Counting Integral Points in Polyhedra when the Dimension is Fixed," Math. Oper. Res. vol. 19, pp. 769-779, 1994.

[26] M. Dyer and R. Kannan, "On Barvinok’s Algorithm for Counting Lattice Points in Fixed Dimension," Math. Oper. Res., vol. 22, pp. 545-549, 1997.

[27] A. Barvinok and J. E. Pommersheim, "An Algorithmic Theory of Lattice Points in Polyhedra," in in: L. J. Billera, A. Björner, C. Greene, R. Simion, and R. P. Stanley, New Perspectives in Algebraic Combinatorics. Cambridge University Press, 1999, vol. 38.

[28] M. E. Dyer, "On counting lattice points in polyhedra," SIAM J. Comput., vol. 20, pp. 695-707, 1991

[29] W. Cook, M. Hartmann, R. Kannan, and C. McDiarmid, "On integer points in polyhedra," Combinatorica, vol. 12, pp. 27-37, 1992.

[30] M. Welleda Baldoni, M. Beck, C. Cochet, and M. Vergne, "Volume computation for polytopes and partition functions for classical root systems," Discrete Comput. Geom., vol. 35, pp. 551-595, 2006.

[31] W. Fulton, Young Tableaux. London Mathematical Society, 1997.

[32] S. Daftuar and P. Hayden, "Quantum state transformations and the Schubert calculus," Ann. Phys., vol. 315, pp. 80-122, 2004.

[33] A. Klyachko, "Quantum marginal problem and representations of the symmetric group," arXiv:quant-ph/0409113, 2004.

[34] M. Christandl, A. W. Harrow, and G. Mitchison, "On Nonzero Kronecker Coefficients and their Consequences for Spectra," Commun. Math. Phys., vol. 270, pp. 575-585, 2007. 
[35] A. W. Harrow, "Applications of coherent classical communication and the Schur transform to quantum information theory," $\mathrm{PhD}$ Thesis, Massachusetts Institute of Technology, 2005.

[36] E. Briand, R. Orellana, and M. Rosas, "Quasipolynomial formulas for the Kronecker coefficients indexed by two two-row shapes (extended abstract)," in Proc. 21st FPSAC. DMTCS, 2009, pp. 241-252.

[37] W. Fulton and J. Harris, Representation Theory: A First Course. Springer, 1991.

[38] P. Bürgisser, M. Christandl, and C. Ikenmeyer, "Even Partitions in Plethysms," J. Algebra, vol. 328, pp. 322-329, 2011.

[39] M. Christandl, N. Schuch, and A. Winter, "Highly Entangled States With Almost No Secrecy," Phys. Rev. Lett., vol. 104, p. 240405, 2010.

[40] L. G. Valiant, "Completeness classes in algebra," in Proc. 11th STOC. ACM, 1979, pp. 249-261.

[41] V. Strassen, "Gaussian elimination is not optimal," Numer. Math., vol. 13, pp. 354-356, 1969.

[42] P. Bürgisser and C. Ikenmeyer, "Geometric complexity theory and tensor rank," in Proc. 43rd STOC. ACM, 2011.

[43] V. Strassen, "Komplexität und Geometrie bilinearer Abbildungen," Jahresber. Deutsch. Math.-Verein., vol. 107, pp. 3-31, 2006.

[44] M. Brion, "Sur l'image de l'application moment," in Séminaire d'Algèbre Paul Dubreil et Marie-Paule Malliavin. Springer, 1987, vol. 1296, pp. 177-192.

[45] P. Bürgisser, M. Christandl, and C. Ikenmeyer, "Nonvanishing of Kronecker coefficients for rectangular shapes," Adv. Math., vol. 227, pp 2082-2091, 2011

[46] S. Kumar, "A Study of the representations supported by the orbit closure of the determinant," arXiv:1109.5996, 2011.

[47] J. A. Grochow and K. Rusek, "Report on "Mathematical Aspects of $\mathbf{P}$ vs. NP and its Variants.,", arXiv:1203.2888, 2012.

[48] A. Okounkov, "Brunn-Minkowski inequality for multiplicities," Invent. Math., vol. 125, pp. 405-413, 1996.

[49] G. J. Heckman, "Projections of Orbits and Asymptotic Behaviour of Multiplicities for Compact Connected Lie Groups," Invent. Math. vol. 67, pp. 333-356, 1982.

[50] V. Guillemin and S. Sternberg, "Geometric Quantization and Multiplicities of Group Representations," Invent. Math., vol. 67, pp. 515-538, 1982.

[51] R. Sjamaar, "Holomorphic slices, symplectic reduction and multiplicities of representations," Ann. Math., vol. 141, pp. 87-129, 1995.

[52] E. Meinrenken, "On Riemann-Roch Formulas for Multiplicities," $J$. Amer. Math. Soc., vol. 9, pp. 373-389, 1996.

[53] E. Meinrenken and R. Sjamaar, "Singular reduction and quantization," Topology, vol. 38, pp. 699-762, 1999.

[54] M. Vergne, "Quantization of Algebraic Cones and Vogan's Conjecture," Pacific J. Math., vol. 182, pp. 113-135, 1998.

[55] C. Teleman, "The quantization conjecture revisited," Ann. Math., vol. 152, pp. 1-43, 2000.

[56] V. Guillemin, E. Lerman, and S. Sternberg, "On the Kostant multiplicity formula," J. Geom. Phys., vol. 5, pp. 721-750, 1988.

[57] — Symplectic Fibrations and Multiplicity Diagrams. Cambridge University Press, 1996.

[58] M. Christandl, B. Doran, S. Kousidis, and M. Walter, "Eigenvalue Distributions of Reduced Density Matrices," arXiv:1204.0741, 2012.

[59] R. W. Carter, G. Segal, and I. G. MacDonald, Lectures on Lie groups and Lie algebras. London Mathematical Society, 1995.

[60] A. Kirillov Jr., An Introduction to Lie Groups and Lie Algebras. Cambridge University Press, 2008.

[61] A. W. Knapp, Lie Groups Beyond an Introduction, 2nd ed. Springer, 2002, vol. 140.

[62] P. Littelmann, "Cones, crystals, and patterns," Transform. Groups, vol. 3, pp. 145-179, 1998.

[63] I. Gelfand and M. Tsetlin, "Finite-dimensional representations of the group of unimodular matrices," in in: I. Gelfand, Collected Papers. Originally appeared in Doklady akademii nauk SSSR, nowaja serija 71 (1950). Springer, 1988, vol. II.

[64] R. Steinberg, "A general Clebsch-Gordan theorem," Bull. Amer. Math. Soc., vol. 67, pp. 406-407, 1961.

[65] J. A. De Loera and T. B. McAllister, "On the computation of ClebschGordan coefficients and the dilation effect," Experiment. Math., vol. 15, pp. 7-19, 2006.

[66] A. D. Berenstein and A. V. Zelevinsky, "Tensor product multiplicities, canonical bases and totally positive varieties," Invent. Math., vol. 143, pp. 77-128, 2001
[67] N. Straumann, "Branching rules and Clebsch-Gordan series of semisimple Lie algebras," Helv. Phys. Acta, vol. 38, pp. 481-498, 1965.

[68] P. Clauss and V. Lochner, "Parametric analysis of polyhedral iteration spaces,” J. VLSI Signal Processing, vol. 19, pp. 179-194, 1998.

[69] S. Verdoolaege, R. Seghir, K. Beyls, V. Loechner, and M. Bruynooghe, "Counting Integer Points in Parametric Polytopes using Barvinok's Rational Functions," Algorithmica, vol. 48, pp. 37-66, 2007.

[70] V. Baldoni and M. Vergne, "Discrete series representations and $K$ multiplicities for $u(p, q)$. user's guide," arXiv:1008.5360, 2010.

[71] J. A. De Loera, B. Dutra, M. Köppe, S. Moreinis, G. Pinto, and J. Wu, "Software for Exact Integration of Polynomials over Polyhedra," arXiv:1108.0117, 2011.

[72] S. Verdoolaege and M. Bruynooghe, "Algorithms for Weighted Counting over Parametric Polytopes: A Survey and a Practical Comparison," in Proc. ITSL. CSREA Press, 2008, pp. 60-66.

[73] R. Hartshorne, Algebraic Geometry. Springer, 1977.

[74] S. Kumar, "Geometry of orbits of permanents and determinants," arXiv:1007:1695, 2010. 